\title{
Characterization and antimicrobial potential of soil actinobacterium TFAl isolated from Talakona forest, Andhra Pradesh
}

\author{
Ponnuswamy Shekar ${ }^{1}$, Manikkam Radhakrishnan ${ }^{2 *}$,Venugopal Gopikrishnan ${ }^{2}$, Arumugam Suresh $^{2}$ \\ ${ }^{1}$ Department of Chemical Engineering, SSN College of Engineering, Chennai-603110, Tamil Nadu, India. \\ ${ }^{2}$ Centre for Drug Discovery and Development, Sathyabama University, Chennai-600119.Tamil Nadu, India.
}

\begin{tabular}{|c|c|}
\hline ARTICLE INFO & ABSTRACT \\
\hline Article history: & \multirow{6}{*}{$\begin{array}{l}\text { The present study reports the characterization and antimicrobial potential of actinobacterial strain TFA1 isolated } \\
\text { from Thalakona forest soil, Andhra Pradesh. Phenotypic, cell wall and molecular characteristics of strain TFA1 } \\
\text { was studied by adopting standard procedures. Strain TFA1 produced powdery colonies on ISP } 2 \text { agar medium } \\
\text { with aerial and substrate mycelium. Cell wall analysis revealed the presence of LL- DAP and glycine but no } \\
\text { sugar. PCR amplification of } 16 \mathrm{~S} \text { rRNA gene of strain TFA } 1 \text { yielded } 1014 \mathrm{bp} \text { sequence. During } 16 \mathrm{~S} \text { rRNA } \\
\text { sequence analysis, strain TFA1 (accession no KP893923) shows } 99 \% \text { similarity to Streptomyces kurssanovii } \\
\text { NBRC } 13192 \text {. In preliminary agar plug method, strain TFA1 showed activity against } S \text {. aureus, B. cereus and } E \text {. } \\
\text { coli. Strain TFA1 produced bioactive metabolites in solid media on the } 3^{\text {rd }} \text { day of fermentation whereas in liquid } \\
\text { media the production of bioactive metabolite was observed on } 5^{\text {th }} \text { day of fermentation. The ethyl acetate extract } \\
\text { of strain TFA1 showed } 14-18 \mathrm{~mm} \text { inhibition against Gram positive and Gram negative bacterial pathogens. }\end{array}$} \\
\hline Received on: $18 / 08 / 2016$ & \\
\hline Accepted on: 16/10/2016 & \\
\hline Available online: 30/03/2017 & \\
\hline Key words: & \\
\hline $\begin{array}{l}\text { Actinobacteria, } \\
\text { characterization, taxonomy, } \\
\text { antimicrobial activity, } \\
\text { fermentation. }\end{array}$ & \\
\hline
\end{tabular}

\section{INTRODUCTION}

Emergence of drug resistance among common microbial pathogens is stern problem that accentuate the development of novel antimicrobials. Microbial resources from unexplored niches made inevitable contribution for antibiotic development process to combat the problem of antibiotic resistance (Harvey et al., 2000). Among the microbial sources, actinobacteria are the most important source of bioactive metabolites and more novel molecules are still on the row to discover from this group of bacteria (Balagurunathan et al., 2010). In particular, diverse actinobacteria from poorly studied unusual environments promises a raise in the prospect of discovering novel compounds (Jose et al., 2013; Yuan et al., 2014).Forest ecosystems are more complex and heterogeneous

\footnotetext{
* Corresponding Author

Manikkam Radhakrishnan, Centre for Drug Discovery and Development, Sathyabama University, Chennai-600119.Tamil Nadu, India.

Email:mrkactinos@yahoo.com
}

environments and they are characterized by a wealthy biological diversity (Lami et al., 1993). Actinobacteria from forest ecosystems including from Western Ghats and Eastern Ghats of India are isolated and investigated for antimicrobial (Radhakrishnan et al., 2014a) and enzymatic activities (Mohanapriya et al., 2011). The present study reports the phenotypic and molecular characterization of actinobacterial strain TFA1 isolated from Talakona forest, Andhra Pradesh and its antimicrobial potential.

\section{MATERIALS AND METHODS}

\section{Description of actinobacterial strain TFA1}

Actinobacterial strain TFA1 (Figure 1) was isolated from the soil sample collected from Talakona forest (Estern Ghats),

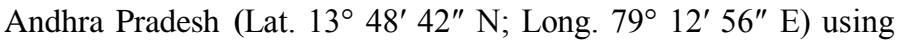
starch casein agar supplemented in the year 2013. Viability of strain TFA1 was maintained on ISP2 agar slants as well as in $30 \%$ glycerol broth at $-20^{\circ} \mathrm{C}$. 


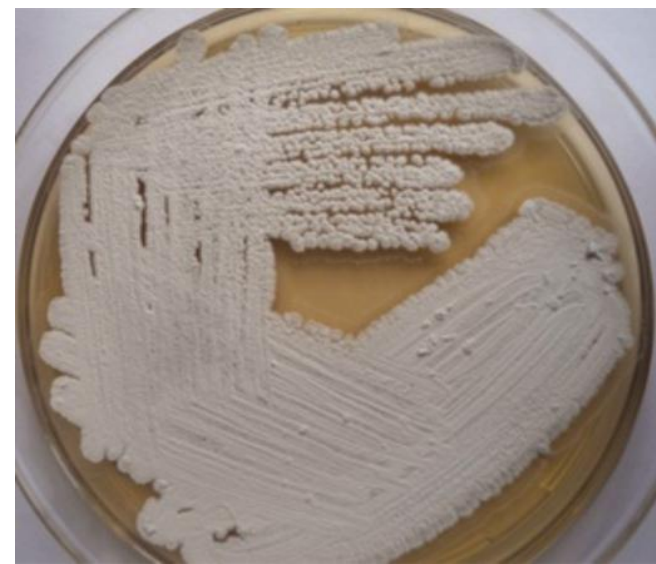

Fig. 1: Growth of actinobacterial strain TFA1 on ISP2 agar

\section{Characterization of actinobacterial strain TFA1 Phenotypic characterization}

The micro morphologicalstructures such as the aerial mycelium, substrate mycelium, mycelial fragmentation were observed under bright field microscope at $40 \mathrm{X}$ magnification (Balagurunathan et al., 2010). The growth characteristics of strain TFA1 on different ISP (International Streptomyces Project) medium were studied ontryptone agar (ISP1), yeast extract-malt extract agar (ISP2), oatmeal agar (ISP3), inorganic salts-starch agar (ISP4), glycerolasparagine agar (ISP5), peptone yeast extractiron agar (ISP6) and tyrosine agar (ISP7) (Shirling and Gottileb, 1966). Effect of different carbon sources (sugars such as glucose, fructose, sucrose, mannitol, inositol, xylose, rhamnose, raffinose, and cellulose) and nitrogen sources (amino acids such as tyrosine, asparagines and glutamine) were studied on the growth of strain TFA1 by adopting standard procedures (Mohanraj et al., 2011). Effect of $\mathrm{pH}(5,7,9 \& 11)$, temperature $\left(20^{\circ} \mathrm{C}, 30^{\circ} \mathrm{C}, 40^{\circ} \mathrm{C}\right.$ and $50^{\circ} \mathrm{C}$ ) on the growth of strain TFA1 was studied using ISP2 agar medium (Radhakrishnan et al., 2013). Isomers of diaminopimelic acid (DAP) and sugars in whole-cell hydrolysates of strain TFA1 were analyzed by adopting the method described by Hasegawa et al.(1983).

\section{Molecular characterization}

The genomic DNA of actinobacterial strain TFA1 was isolated using the InstaGeneTM Matrix Genomic DNA isolation kit. Mycelial growth of actinobacterial strain was picked from ISP2 agar plates and suspended in $1 \mathrm{ml}$ of sterile water in a microfuge tube. The content was centrifuged for 1 minute at $10,000 \mathrm{rpm}$ to remove the supernatant and the cells were pelleted. Then $200 \mu \mathrm{l}$ of Insta Gene matrix was added to the pellet and incubate at $56{ }^{\circ} \mathrm{C}$ for 15 minutes and vortexed at high speed for 10s (Delalibera et al., 2007). The mixture was heated in a boiling water bath for 10 minutes and then centrifuged at high speed to separate the matrix. Twenty microliters of the resulting DNA was used in a 50- $\mu 1$ PCR reaction to construct a $16 \mathrm{~S}$ rRNAgene library. General primers for bacteria $27 \mathrm{~F}$ (5'AGAGTTTGATCCTGGCTCAG-3') and 1492R (5'TACCTTGTTACGACTT-3') were used to amplify 16S rRNA genes from DNA extracted from the actinobacterial strain TFA1. Final concentrations for 50- $\mu$ l PCR reactions were as follows: $2 \mu 1$ diluted DNA (10-100 ng) (or $20 \mu \mathrm{l}$ for DNA extracted using InstaGeneTM Matrix), $0.2 \mu \mathrm{M}$ of each primer, $0.2 \mathrm{mM}$ dNTPs, 5 units of Taq polymerase and $1 \mathrm{X}$ Taq polymerase buffer. The reaction conditions were: $94^{\circ} \mathrm{C}$ for $3 \mathrm{~min}, 35$ cycles at $94{ }^{\circ} \mathrm{C}$ for 30 $\mathrm{s}, 55^{\circ} \mathrm{C}$ for $1.5 \mathrm{~min}$, and $72{ }^{\circ} \mathrm{C}$ for $2.5 \mathrm{~min}$, and a final extension at $72^{\circ} \mathrm{C}$ for $5 \mathrm{~min}$. By using Montage PCR clean up kit (Millipore), the unincorporated PCR primers and dNTPs have been removed.

Single-pass sequencing was performed on each template using 518F/800R primers (518F 5'-CCAGCAGCCGCGGTAATACG-3' and 800R 5'- TACCAGGGTATCTAATCC-3'). Sequencing reactions were performed using a ABI PRISM ${ }^{\circledR}$ BigDyeTM Terminator Cycle Sequencing Kits with AmpliTaq ${ }^{\circledR}$ DNA polymerase (Applied Biosystems).

The 16S rRNA sequence of actinobacterial strains were subjected to BLAST similarity search tool. By comparing the sequence similarity with non redundant database of nucleotide sequences deposited at NCBI (http://www.ncbi.nlm.nih.gov/) through BLAST program, closely related homologs were identified. The program MUSCLE 3.7 was used for to process the multiple alignments of sequences. The stability of relationship was admittance by performing bootstrap analysis for 1000 replicates. Divergence times for all branching points in the topology were calculated with the Real Time method using the branch lengths contained in the inferred tree. Bars around each node represent 95\% confidence intervals were computed (Tamura et al., 2011). All positions containing gaps and missing data were eliminated. The evolutionary history was inferred by using the NeighborJoining method in the MEGA 6 software. Identification of species through sequence similarity between query sequence and reference sequence based on criteria, for $99 \%$ or above similarity assigned to reference species, 99-95\% similarity assigned to corresponding genus, less than 95\%, assigned to corresponding family (Bosshard et al., 2003). The $16 \mathrm{~S}$ rRNA sequence of potential actinobacterial cultures were submitted to Genbank to get the accession number.

\section{Antimicrobial activity of actinobacterial strain TFA1}

Antimicrobial activity of strain TFA1 was studied by adopting agar plug method against Staphylococcus aureus, Bacillus cereus, Escherichia coli, Pseudomonas aeruginosa and Candida albicans (Radhakrishnan et al., 2014b). Effect of solid and liquid medium on bioactive metabolite production from the strain TFA1 was studied using ISP2 medium. Antimicrobial activity was tested for every 24 hours against $S$. aureus. Crude bioactive metabolite from the agar medium was extracted using ethyl acetate and tested for antimicrobial activity by adopting disc diffusion method (Radhakrishnan et al., 2014b).

\section{RESULTS AND DISCUSSION}

\section{Phenotypic characteristics of strain TFA1}

The characterization of a strain is a key element in systematics of prokaryotes including actinobacteria. Although 
various new methodologies have been developed over the past 100 years, both the newer methodologies and those considered to be traditional remain key elements in determining whether the strain belong to known taxon or constitute a novel one (Tindall et al., 2010). Actinobacterial classification was originally based largely on morphological observations and physiological characteristics (Shirling and Gottileb, 1966; Nonomura, 1974). The advent of chemotaxonomic criteria has provided reproducible and reliable tools to identify the genera at genus level (Labeda, 1990). In the present study, strain TFA1 shows good growth on all ISP medium except in ISP1 medium. Under bright field microscopic observation, strain TFA1 showed the presence of rectus flexible type aerial mycelium and non fragmented substrate mycelium (Figure 2).

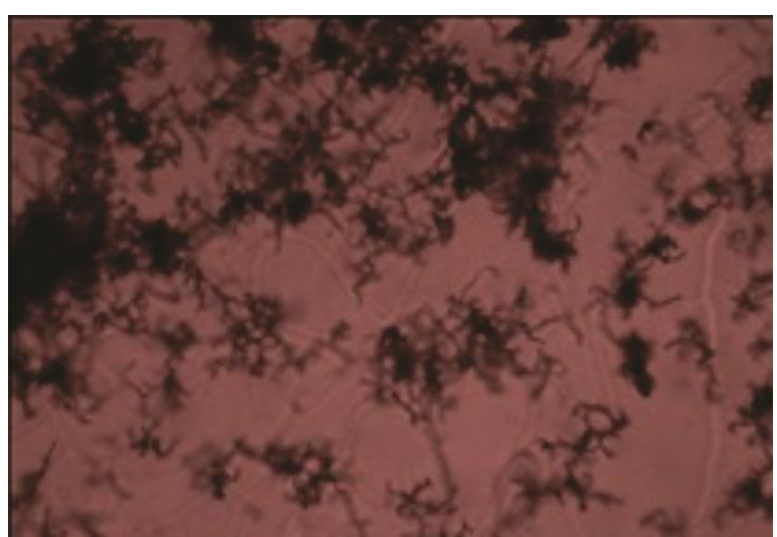

Fig. 2: Micromorphology of actinobacterial strain TFA1 undeerbright field microscope.

Strain TFA1 showed good growth on all the ISP media tested except ISP1 and ISP3 and it also utilized wide range of sugars, pH $7 \& 9$ and temperature $30^{\circ} \mathrm{C}$ (Table 1). The whole cellhydrolysate of isolate TFA1 were rich diaminopimelic acid (DAP), with no characteristic sugar indicates chemotype I cell wall.The results of phenotypic characterisation revealed that the actinobacterial strain TFA1 belongs to a species of the genus Streptomyces. But it is not adequate in itself to differentiate between genera.

Table 1: Phenotypic characteristics of actinobacterial strain TFA1

\begin{tabular}{cc} 
Table 1: Phenotypic characteristics of actinobacterial strain TFA1. \\
\hline Characteristics & Strain TFA1 \\
Micromorphology & Present \\
Aerial mycelium & Present \\
Substrate mycelium & Non-fragmented \\
Fragmentation & $30-40$ \\
Number of spores & \\
Cultural characteristics & Brown \\
Aerial mycelialcolour & Absent \\
Reverseside pigment & Absent \\
Diffusible pigment & Moderate \\
ISP1 (Tryptone agar) & Good \\
ISP3 (Oatmeal agar) & Good \\
ISP2 (Yeast extract malt extract agar) & Good \\
ISP4 (Inorganic salts- starch agar) & Good \\
ISP5 (Glycerol aspargine agar) & Good \\
ISP6 (Peptone Yeast extract ironagar) & Good \\
ISP7 (Tyrosine agar) & \\
Utilization of &
\end{tabular}

\begin{tabular}{cc}
\hline Glucose & Good \\
Arabinose & Good \\
Sucrose & Good \\
Xylose & Moderate \\
Inositol & Good \\
Mannitol & Good \\
Fructose & Moderate \\
Rhamnose & Moderate \\
Raffinose & Moderate \\
Cellulose & Good \\
Growth at & \\
pH 5 & Moderate \\
pH 7 & Good \\
pH 9 & Good \\
pH 11 & Poor \\
$20^{\circ} \mathrm{C}$ & Poor \\
$30^{\circ} \mathrm{C}$ & Good \\
$40^{\circ} \mathrm{C}$ & Moderate \\
$50^{\circ} \mathrm{C}$ & No growth \\
\hline
\end{tabular}

\section{Molecular characterization of strain TFA1}

The analysis of $16 \mathrm{~S}$ rRNA gene sequence has also revealed that the strain TFA1 belongs to a species of the genus Streptomyces. The PCR amplification the 16S rRNA gene of actinobacterial strain TFA1 yielded 1014 base pairs. During BLAST analysis the $16 \mathrm{~S}$ rRNA gene sequence of strain TFA1 showed $99 \%$ similarity to the gene sequence of Streptomyces kurssanovii NBRC 13192 published in GenBank. The 16S rRNA gene sequence of strain TFA1 was published in GenBank with the accession no KP893923.

The phylogenetic relatedness of strain TFA1 with its closely related species was given in figure 3 .

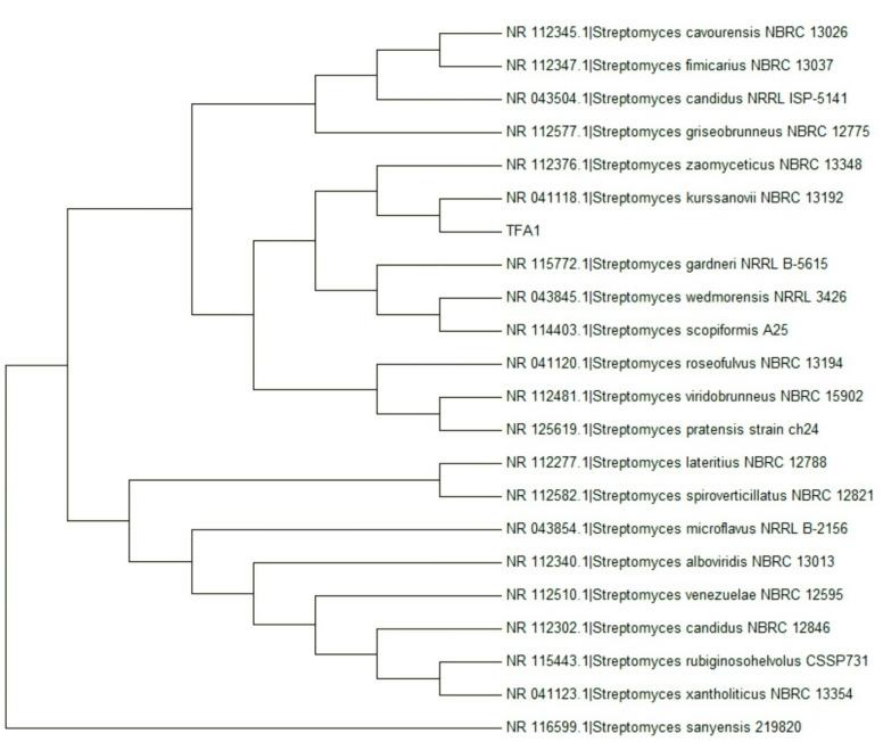

Fig. 3: Phylogenetic tree showing relationship of the Streptomyces species TFA1 with closely related species of the genus Streptomyces inferred from aligned sequence of $16 \mathrm{~S}$ rRNA gene. The optimal tree with sum of branch length $=0.95$ is shown. The percentage of replicate trees in which the associated taxa clustered together in the bootstrap test ( 100 replicates) is shown next to the branches. The evolutionary distances were computed using the p-distance method and are in the units of the number of base differences per site. There were a total of 1014 positions in the final dataset. 


\section{Antimicrobial potential of strain TFA1}

In agar plug method, strain TFA1 inhibited $S$. aureus $(15$ $\mathrm{mm})$, B. cereus $(14 \mathrm{~mm})$ and $E$. coli $(10 \mathrm{~mm})$. During submerged and agar surface fermentation process, strain TFA1 produced bioactive metabolites in submerged culture on $5^{\text {th }}$ day of incubation whereas in solid culture bioactive metabolite production was observed even on $3^{\text {rd }}$ of incubation. Effect of incubation period and medium consistency on bioactive metabolite production from the strain TFA1 was given in figure 4 .

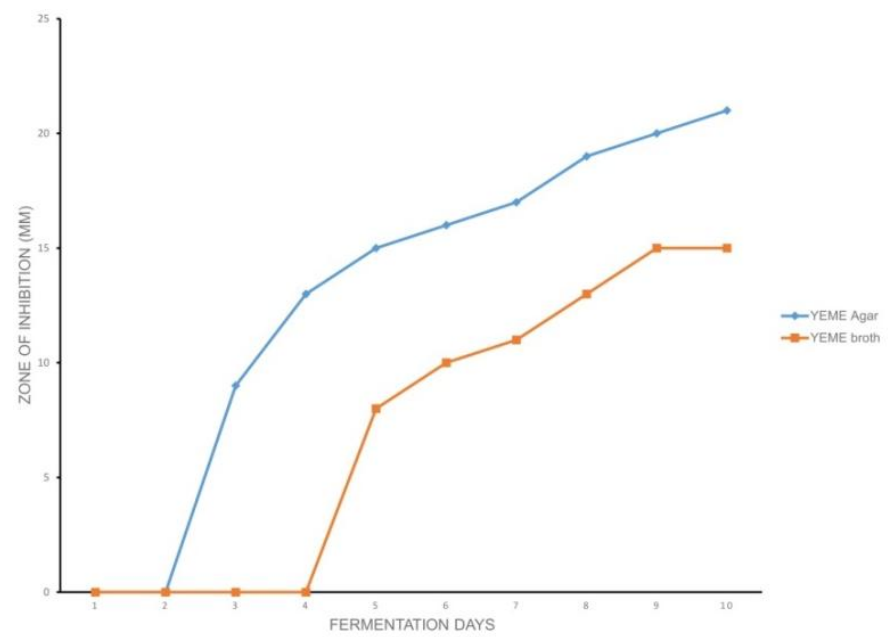

Fig. 4: Effect of incubation period and medium consistency on bioactive metabolite production by the strain TFA1

The morphology of actinobacterial mycelium is well suited for invasive growth on solid medium (Barrios-Gonzlez and Mejia, 1996). The hyphal mode of growth gives the filamentous organism the power to penetrate into the solid substrates. This also gives them a major advantage over unicellular microorganisms for the colonisation of the substrate and the utilisation of the available nutrients.

Therefore, SSF technology can be exploited as an alternative, allowing better oxygen circulation, especially when large quantities of secondary metabolites are required to be produced in short fermentation periods with minimal expenditure of media and downstream processing (El-Naggar et al., 2009). In the present study, the bioactive metabolite from strain TFA1 was produced by agar surface fermentation, a variant of solid state fermentation. There are many reports on antibiotic production from actinobacteria through SSF using agricultural substrates (ElNaggar et al., 2009). Further, studies on solid state fermentation using agricultural substrates may results in the economic production of bioactive compound from the potential actinobacterial strain TFA1.

Majority of the actinobacterial metabolites are extracellular in nature and they are extracted using the medium polar solvent ethyl acetate (Selvameenal et al., 2009). The ethyl acetate extract from the strain TFA1 showed $18 \mathrm{~mm}$ inhibition against $S$. aureus and B. cereus and $14 \mathrm{~mm}$ inhibition against $E$. coli in disc diffusion method. This result indicates the medium polar nature of the active compound produced by the actinobacterial strain TFA1.Further production and isolation of active metabolites from the actinobacterial strain TFA1 is in progress.

\section{REFERENCES}

Balagurunathan R, Radhakrishnan M, Somasundaram ST. Lglutaminase producing actinomycetes from marine sediments: selective isolation, semi quantitative assay and characterization of potential strain. Australian J Basic Appl Sci, 2010; 4(5): 698-705.

Barrios-Gonzlez J, Meija A. Production of secondary metabolites by solid- state fermentation. Biotechnol. Ann. Rev, 1996; 2: 85-121.

Bosshard PP, Zbinden R, Abels S, Boddinghaus B, Altwegg M, Bottger EC. 16S rRNA gene sequencing versus the API 20 NE system and the VITEK 2 ID-GNB card for identification of non fermenting Gramnegative bacteria in the clinical laboratory. J Clinical Microbiol, 2006; 44: 1359-1366.

Delalibera I, Vasanthakumar A, Burwitz BJ, Schloss PD, Klepzig KD, Handelsman J, Kenneth FR, Composition of the bacterial community in the gut of the pine engraver, Ipspini(Say) (Coleoptera) colonizing red pine. Symbiosis, 2007; 43:97-104.

El-Naggar E, Moustafa Y, Swamy A, Assar E, Gawad MA. Solid state fermentation for the production of meroparamycin by Streptomyces sp. Strain MAR01. J Microbiol. Biotechnol, 2009;19(5): 468-473.

Harvey A, Strategies for discovering drugs from previously unexplored natural products. Research focus Reviews, 2000;5: 294-300.

Hasegawa T, Takizawa M, Tanida S. A Rapid Analysis for Chemical Grouping of Aerobic Actinomycetes. J. Gen. Appl. Microbiol, 1983;29:319-322.

Jose PA, Jebakumar SRD, Diverse actinomycetes from Indian coastal solar salterns - a resource for antimicrobial screening. J. Pure Appl. Microbiol, 2013;7: 2569-2575.

Labeda DP, Shearer MC. Isolation of Actinomycetes for biotechnological applications. In:Labeda DP (ed). Isolation of Biotechnological Organisms from Nature. Labeda, D. P. (ed.). McGrawHill Publishing Company, New York. 1990;pp. 1-19.

Lami N, Osada H, Screening of biologically active secondary metabolites from Zarian (Africa) microorganisms. Riken Review. 1993; 3:15-16.

Mohanapriya P, Radhakrishnan M, Balagurunathan R. Production and optimization of L-asparaginase from Streptomyces $s p$ (TA22) isolated from Western Ghats, India. J Chem. Pharm. Res, 2011; 3: 618-624.

Mohanraj D, Bharathi S, Radhakrishnan M, Balagurunathan R. Bioprospecting of actinobacteria from Yelagiri hills with special reference to antibacterial activity. J Chem. Pharm. Res, 2011;3:439-446.

Nonomura H, Key for classification and identification of 458 species of the Streptomycetes included in ISP. J Ferment Technol. 1974; 52: 78-92.

Radhakrishnan M,Gopikrishnan V, Balaji S, Balagurunathan R, Vanaja Kumar. Bioprospecting of actinomycetes from certain less explored ecosystems active against Mycobacterium tuberculosis and other non-mycobacterial pathogens. International Scholarly Research Notices. 2014a; Article ID 812974, 9 pages.

Radhakrishnan M, Pazhanimurugan R, Gopikrishnan V, Balagurunathan R, Vanaja Kumar. Streptomycessp D25 isolated from Thar Desert soil, Rajasthan producing pigmented antituberculosis compound only in solid culture.J Pure Appl Microbio. 2014b; 8(1); 333-337. 
Selvameenal L, Radhakrishnan M, Balagurunathan R, Antibiotic Pigment from Desert Soil Actinomycetes. II. Biological activity, purification and chemical screening. Indian J Pharm. Sci. 2009; 71(4): 499-504.

Shirling EB, Gottileb D, Methods for characterization of Streptomyces species.Int J Syst Evol Microbiol. 1966; 6: 313-340.

Tamura K, Peterson D, Peterson N, Stecher G, Nei M, Kumar S, MEGA5: molecular evolutionary genetics analysis using maximum likelihood, evolutionary distance, and maximum parsimony methods. Mol Biol Evol. 2011; 28: 2731-2739.

Tindall BJ, Rossello R. Busse HJ, Ludwig W, Kampfer P, Notes on the characterization of prokaryote strains for taxonomic purposes. Int $\mathrm{J}$ Syst Evol Microbiol. 2010; 60: 249-266.

Yuan M, Yu Y, Li HR, Dong N, Zhang XH, Phylogenetic diversity and biological activity of actinobacteria isolated from the chukchi shelf marine sediments in the Arctic Ocean. Mar. Drugs. 2014; 212: 1281-1297.

\section{How to cite this article:}

Shekar P, Radhakrishnan M, Gopikrishnan V, Suresh A. Characterization and antimicrobial potential of soil actinobacterium TFA1 isolated from Talakona forest, Andhra Pradesh. J App Pharm Sci, 2017; 7 (03): 202-206. 